\title{
Advances in Epigenetic Cancer Therapeutics
}

Christopher Hillyar $^{1}$, Kathrine S. Rallis ${ }^{1}$, Jajini Varghese ${ }^{2}$

1. Oncology, Barts and the London School of Medicine and Dentistry, Queen Mary University of London, London, GBR 2. Breast and Plastic Surgery, University College London Institute of Surgery and Interventional Science \& Royal Free NHS Trust, London, GBR

Corresponding author: Christopher Hillyar, c.r.t.hillyar@smd17.qmul.ac.uk

\begin{abstract}
Cancer has traditionally been hailed a genetic disease, dictated by successive genetic aberrations which alter gene expression. Yet, recent advances in molecular sequencing technologies, enabling the characterisation of cancer patient phenotypes on a large scale, have highlighted epigenetic changes as a hallmark of cancer. Epigenetic modifications, including DNA methylation and demethylation and histone modifications, have been found to play a key role in the pathogenesis of a wide variety of cancers through the regulation of chromatin state, gene expression and other nuclear events. Targeting epigenetic aberrations offers remarkable promise as a potential anti-cancer therapy given the reversible nature of epigenetic changes. Hence, epigenetic therapy has emerged as a rapidly advancing field of cancer research. A plethora of epigenetic therapies which inhibit enzymes of post-translational histone modifications, so-called 'writers', 'erasers' and 'readers', have been developed, with several epigenetic inhibitor agents approved for use in routine clinical practice. Epigenetic therapeutics inhibit the methylation or demethylation and acetylation or deacetylation of DNA and histone proteins. Their targets include writers (DNA methyltransferases [DNMT], histone acetyltransferases [HAT] and histone deacetylases [HDAC]) and erasers (histone demethylases $[\mathrm{HDM}]$ and histone methylases [HMT]). With new epigenetic mechanisms increasingly being elucidated, a vast array of targets and therapeutics have been brought to the fore. This review discusses recent advances in cancer epigenetics with a focus on molecular targets and mechanisms of action of epigenetic cancer therapeutics.
\end{abstract}

Received 09/06/2020 Review began 09/07/2020 Review ended 11/14/2020 Published 11/27/2020

๑) Copyright 2020

Hillyar et al. This is an open access article distributed under the terms of the Creative Commons Attribution License CC-BY 4.0., which permits unrestricted use, distribution, and reproduction in any medium, provided the original author and source are credited.
Categories: Genetics, Oncology, Healthcare Technology

Keywords: cancer, epigenetics, dna methylation, histone modifications, inhibitors, histone methyltransferase, histone demethylase, histone acetyltransferase, histone deacetylase

\section{Introduction And Background}

Cancer is a leading cause of avoidable premature death in the United Kingdom (UK) [1]. One in two people born after 1960 can expect to be diagnosed with cancer in their lifetime, with 367,167 new cancer cases and 164,901 cancer deaths occurring nationally each year in the UK [2]. Although risk factors vary between cancer types, cancer is primarily a genetic disease arising through successive genetic aberrations. Through projects such as the International Cancer Genome Consortium and The Cancer Genome Atlas Program, the consequences of cancer gene mutations are emerging. However, the role of the epigenome in reshaping gene expression profiles has also come to light (Figure 1). This review aims to highlight recent advances in our understanding of cancer epigenetics and the key targets for and mechanisms of epigenetic cancer therapeutics.

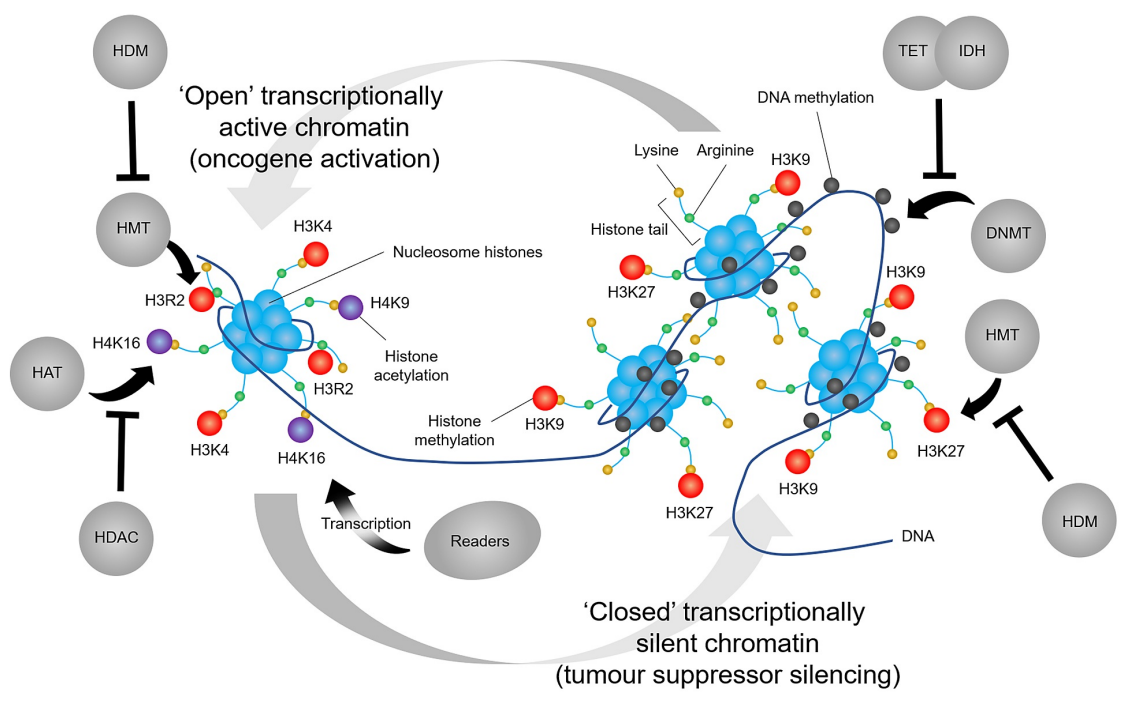

FIGURE 1: The antagonistic role of writers and erasers changes 


\section{chromatin structure and regulates gene transcription, activating and} silencing oncogenes and tumour suppressors

In the nucleus, the human genome is highly organised into a tightly packaged DNA-protein complex called chromatin, which consists of basic units called nucleosomes. Nucleosomes comprise 147 base-pairs of DNA wrapped around an octamer of histone proteins (two H2A-H2B dimers and one H3-H4 tetramer), which is covalently modified by enzymes called 'writers', 'erasers' and 'readers'. These epigenetic machines, which are the target of different anti-cancer drugs, are responsible for maintaining, removing and binding to epigenetic modifications to direct epigenetic effects. Certain families of writers and erasers act antagonistically to orchestrate changes in transcriptionally active euchromatin and transcriptionally silent heterochromatin

Adapted from [3].

DNMT, DNA methyltransferase; HAT, histone acetyltransferase; HDAC, histone deacetylase; HDM, histone demethylase; HDMI, histone demethylase inhibitor; IDH, isocitrate dehydrogenase; TET, ten-eleven translocation protein

\section{Review}

Table 1 provides a list of select epigenetic cancer therapeutics under development. Despite advances in cancer epigenetics, only two epigenetic cancer therapeutics are currently recommended for the treatment of cancer patients by the National Institute of Health and Care Excellence (NICE) in the UK. These include the DNA methyltransferase (DNMT) inhibitor 5'-azacytidine (Aza; Vidaza $\left.{ }^{\circledR}\right)$ for myelodysplastic syndrome and certain types of leukaemia [4], and the histone deacetylase (HDAC) inhibitor panobinostat (LBH589) for multiple myeloma [5]. In addition, the enhancer of zeste homolog 2 (EZH2) inhibitor tazemetostat (EPZ6348) has been granted orphan drug approval in Europe for large B-cell lymphoma by the European Medicines Agency [6]. In the United States, however, nine epigenetic therapeutics have been approved by the U.S. Food and Drug Administration, including two DNMT inhibitors (DNMTIs), four HDAC inhibitors, two isocitrate dehydrogenase (IDH) inhibitors, and, recently, the EZH2 inhibitor tazemetostat [7].

\begin{tabular}{|c|c|c|c|}
\hline Group & Target & Compound & Cancer \\
\hline \multirow{4}{*}{$\begin{array}{l}\text { DNMT } \\
\text { inhibitor }\end{array}$} & DNMT1 & $\begin{array}{l}\text { 5-aza-2'-deoxycytidine (5- } \\
\text { aza-CdR; decitabine, } \\
\text { Dacogen } \circledast)\end{array}$ & Myelodysplastic syndrome, acute myeloid leukaemia \\
\hline & DNMT1 & $\begin{array}{l}\text { 5-azacytidine (5-aza-CR; } \\
\text { Aza; Vidaza®) }\end{array}$ & Myelodysplastic syndrome, acute myeloid leukaemia \\
\hline & $\begin{array}{l}\text { DNMT1, DNMT3, } \\
\text { cytidine } \\
\text { deaminase }\end{array}$ & $\begin{array}{l}\text { Zebularine (NSC309132; 4- } \\
\text { deoxyuridine) }\end{array}$ & Hematologic and solid cancers \\
\hline & DNMT1 & Guadecitabine (SGI-110) & Hematologic and solid cancers \\
\hline \multirow{4}{*}{$\begin{array}{l}\text { HAT } \\
\text { inhibitor }\end{array}$} & p300 & C646 & Prostate cancer \\
\hline & p300, CBP & Curcumin & Multiple myeloma, breast cancer, pancreatic cancer \\
\hline & p300, PCAF & Anacardic acid & NA \\
\hline & p300, PCAF & Garcinol & NA \\
\hline \multirow{7}{*}{ inhibitor } & $\begin{array}{l}\text { Class I, II, IV } \\
\text { HDAC }\end{array}$ & Sulforaphane (SFN) & Leukaemia, colorectal cancer, prostate cancer, other solid tumours \\
\hline & Class I HDAC & Domatinostat (4SC-202) & Leukaemia, colorectal cancer \\
\hline & $\begin{array}{l}\text { Class I, II, IV } \\
\text { HDAC }\end{array}$ & $\begin{array}{l}\text { Resminostat (4SC-201, } \\
\text { RAS2410) }\end{array}$ & $\begin{array}{l}\text { Leukaemia, colorectal cancer, head and neck cancer, hepatocellular } \\
\text { carcinoma }\end{array}$ \\
\hline & $\begin{array}{l}\text { Class I, II, IV } \\
\text { HDAC }\end{array}$ & Panobinostat (LBH589) & $\begin{array}{l}\text { Cutaneous T-cell lymphoma, Hodgkin's lymphoma, breast cancer, head } \\
\text { and neck cancer, prostate cancer, colorectal cancer, thyroid cancer }\end{array}$ \\
\hline & $\begin{array}{l}\text { Class I, II, IV } \\
\text { HDAC }\end{array}$ & Vorinostat (SAHA, Zolinza $®)$ & $\begin{array}{l}\text { Cutaneous } 1 \text {-cell lymphoma, leukaemı, prostate cancer, bladder cancer, } \\
\text { breast cancer }\end{array}$ \\
\hline & $\begin{array}{l}\text { Class I, II, IV } \\
\text { HDAC }\end{array}$ & $\begin{array}{l}\text { Romidepsin (depsipeptide, } \\
\text { FK228) }\end{array}$ & Cutaneous T-cell lymphoma \\
\hline & $\begin{array}{l}\text { Class I HDAC1, } \\
9,11\end{array}$ & $\begin{array}{l}\text { Entinostat (MS-275, SNDX- } \\
\text { 275) }\end{array}$ & Hodgkin lymphoma, kidney cancer, breast cancer \\
\hline
\end{tabular}




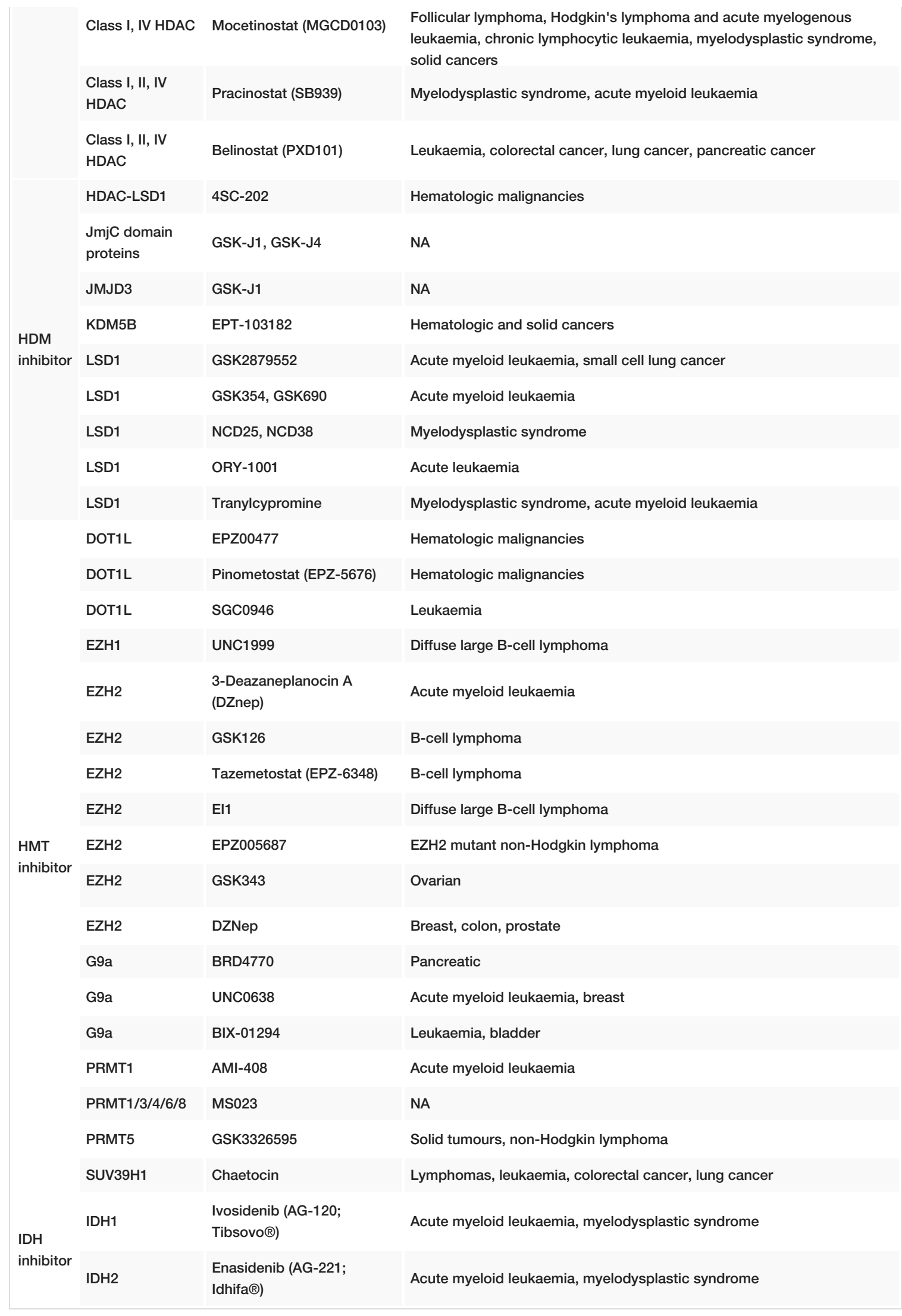

\section{TABLE 1: Select epigenetic cancer therapeutics}

Adapted from [8].

DNMT, DNA methyltransferase; HAT, histone acetyltransferase; HDAC, histone deacetylase; HDM, histone demethylase; HMT, histone methyltransferase; IDH, isocitrate dehydrogenase; DNMT1, DNA (cytosine-5)-methyltransferase 1; DNMT3, DNA (cytosine-5)-methyltransferase 3; p300, E1A binding protein p300; CBP, CREB-binding protein; PCAF, P300/CBP-associated factor; LSD1, lysine-specific histone demethylase 1A; 


\section{Cureus}

JMJD3, histone H3 lysine 27 (H3K27) demethylase KDM6B; KDM5B, lysine-specific demethylase 5B (also known as histone demethylase JARID1B); DOT1L, histone H3K79 methyltransferase; EZH1, enhancer of zeste homolog 1 (histone-lysine N-methyltransferase); EZH2, enhancer of zeste homolog 2 (histone-lysine N-methyltransferase); G9a, protein-lysine methyltransferase; PRMT1/3/4/5/6/8, protein arginine N-methyltransferase 1/3/4/5/6/8; SUV39H1, histone-lysine N-methyltransferase

\section{Therapeutics targeting DNA methylation and demethylation}

The writers and erasers responsible for regulating DNA methylation include DNMTs and ten-eleven translocation (TET) proteins. DNMTs (DNMT1/2/3) transfer methyl groups from the methyl donor Sadenosyl methionine (SAM) to the 5' position of cytosine, forming 5-methylcytosine ( $5 \mathrm{mC})$. Although deposition of $5 \mathrm{mC}$ in the gene promoter is recognised as a cause of gene repression, gene activation may result from $5 \mathrm{mC}$ deposition in hypermethylated promoters and enhancer elements [9]. Generally, however, DNA methylation of ' $\mathrm{CpG}$ islands' (CGIs), which are highly concentrated clusters of cytosine-phosphateguanosine $(\mathrm{CpG})$ dinucleotides, restricts binding of transcription factors at promoters, while promoter CGI hypomethylation allows transcription factor binding and gene activation.

Dysregulation of these processes occurs in cancer. A number of anti-cancer drugs have been developed that target DNMTs. DNMTIs, such as the cytosine analogues Aza (Vidaza) and 5-aza-2'-deoxycytidine (decitabine; Dacogen ${ }^{\circledR}$ ), and the second-generation hypomethylating prodrug SGI-110 (guadecitabine) are classed as DNA hypomethylating agents. Their mechanism of action involves incorporation into DNA and irreversible binding to DNMT1, leading to DNA-DNMT1 adduct formation, DNMT1 degradation and, consequently, DNA demethylation (Figure 2). Aza also incorporates into RNA, more efficiently than DNA, following phosphorylation by uridine-cytidine kinase into triphosphates, resulting in polyribosome disassembly, defective methylation and acceptor function of transfer RNA and translation inhibition $[10,11]$. DNMTIs reduce aberrant hypomethylation and reactivate of silenced genes, thus restoring the function of tumour suppressor genes and DNA repair genes. In addition to the reactivation of tumour suppressor genes, DNMTIs enhance tumour immunogenicity through the upregulation of major histocompatibility complex (MHC) class I, leading to the recruitment of macrophages, natural killer (NK) cells and CD8+ T cells that secrete a variety of chemotactic and cytotoxic cytokines [12].

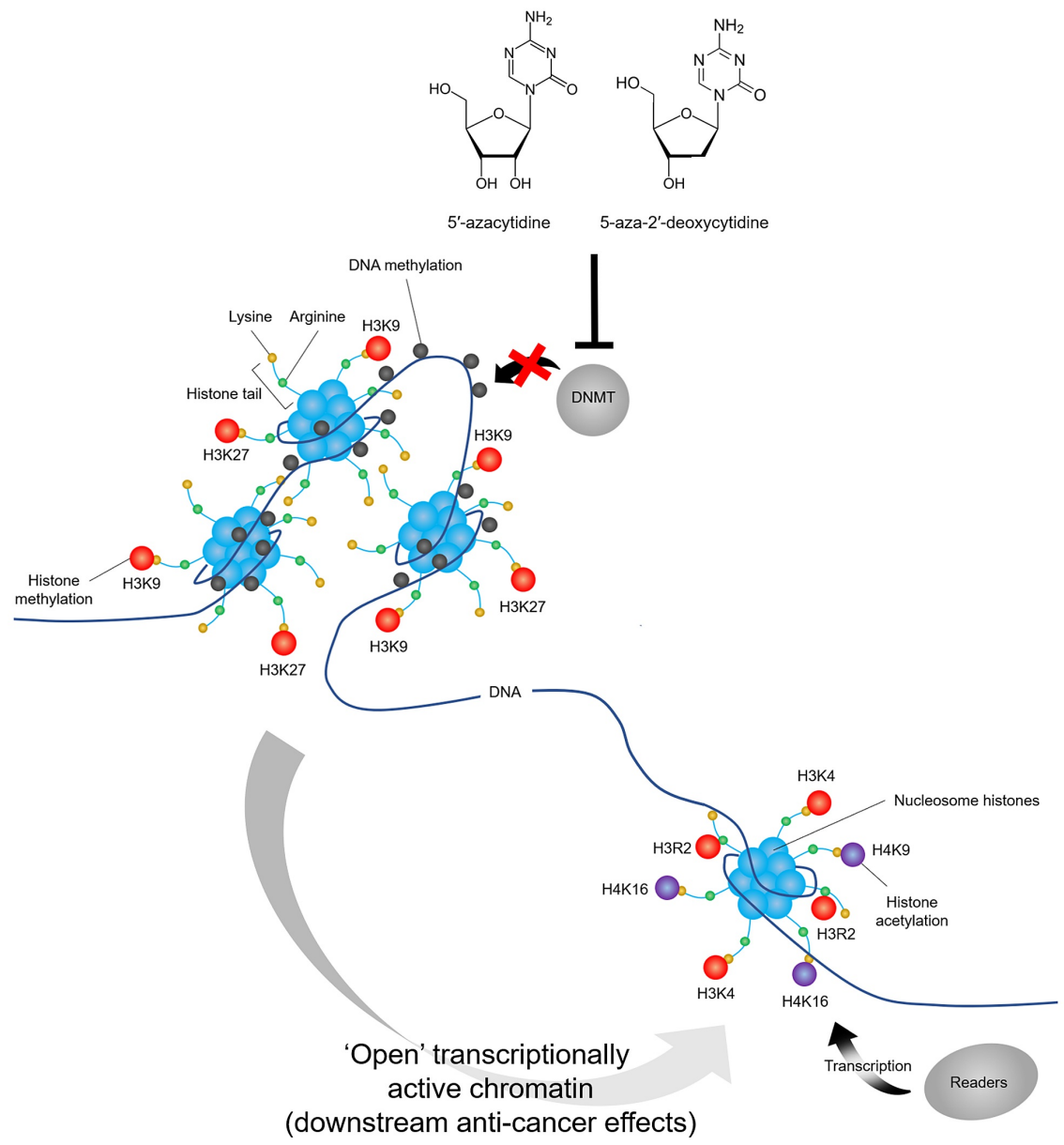

FIGURE 2: Mechanism of action of DNA methyltransferase inhibitors

5 '-azacytidine (Aza; Vidaza) and 5-aza-2'-deoxycytidine (decitabine; Dacogen) incorporate into DNA and irreversibly bind to DNMT1, leading to DNA-DNMT1 adduct formation, DNMT1 degradation and, 


\section{Cureus}

consequently, DNA demethylation and thus leading to the re-expression of tumour suppressor genes with downstream anti-cancer effects.

DNMT1, DNA methyltranferase-1

In contrast to DNMTs, erasers, such as TET proteins, demethylate DNA. TET proteins include a family of enzymes (TET1/2/3) that utilise Fe(II) and 2-oxoglutarate (alpha-ketoglutarate) as cofactors to oxidise $5 \mathrm{mC}$ to 5 -hydroxymethylcytosine $(5 \mathrm{hmC}) .5 \mathrm{hmC}$ formation alters transcriptional activation of gene expression. TET proteins also convert $5 \mathrm{hmC}$ to 5 -formylcytosine $(5 \mathrm{fC})$ or 5 -carboxylcytosine $(5 \mathrm{caC})$ before an enzyme called thymine-DNA glycosylase excises $5 \mathrm{fC}$ and $5 \mathrm{caC}$ from DNA. TET protein function is supported by IDH enzymes, which provide the essential 2-oxoglutarate cofactors through conversion of isocitrate to 2oxoglutarate, and, together, these mechanisms complete DNA demethylation [13].

IDH1 and IDH2 mutations are present across several cancers including in 5-16\% and 6-19\% of acute myeloid leukaemia (AML), respectively [14]. TET2 loss-of-function mutations are present in 16\% of AML [15]. Anticancer drugs that alter IDH and TET enzyme activity have been developed, including the IDH inhibitors AG-120 (ivosidenib) and AG-221 (enasidenib). These drugs inhibit IDH1/2-mediated conversion of $\alpha$ ketoglutarate to 2-hydroxygluterate [16]. 2-hydroxygluterate is an oncometabolite that competitively inhibits TET enzymes [17], which leads to loss of $5 \mathrm{hmC}$ and is associated with carcinogenesis across several malignancies (Figure 3) [18]. IDH inhibitors induce primary AML cell differentiation [19]. In clinical trials, ivosidenib induced durable remission [20], while enasidenib was found to be well tolerated and induced responses in AML patients [21].

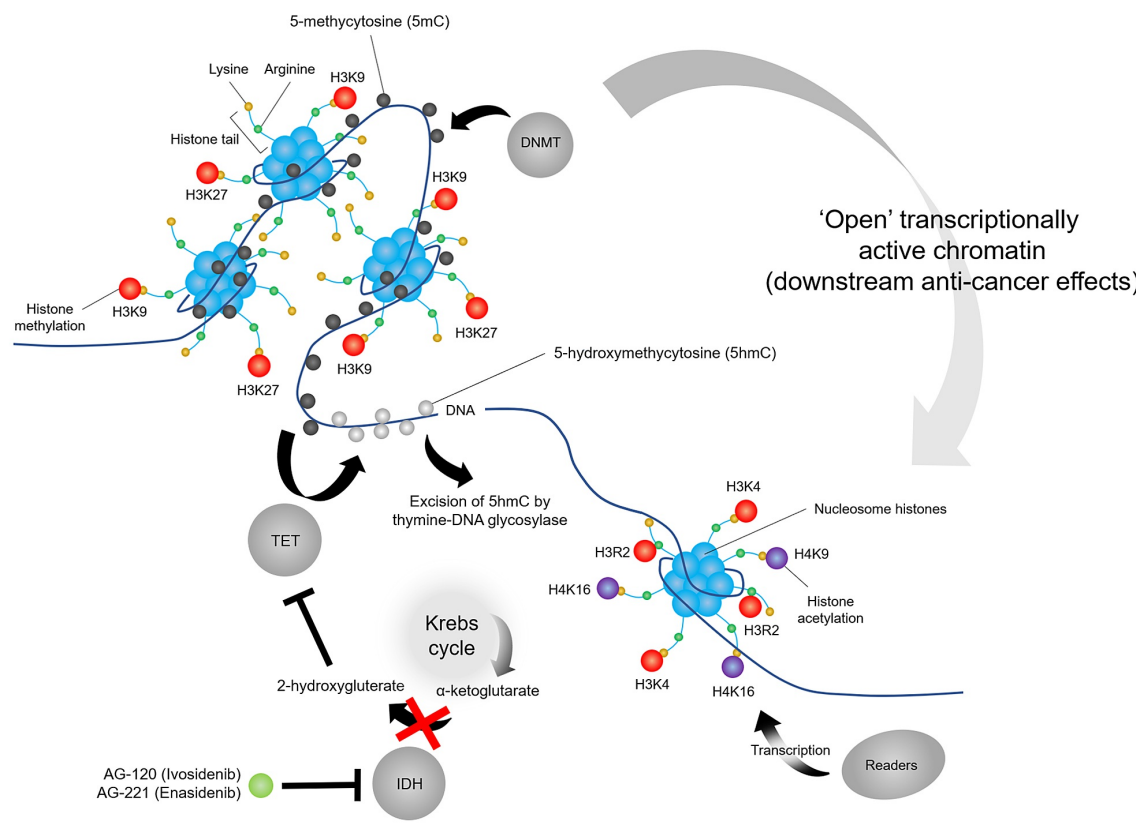

\section{FIGURE 3: Mechanism of action of IDH inhibitors}

DNMT enzymes methylate cytosine bases (5-methylcytosine [ $5 \mathrm{mC}]$ ), while TET proteins sequentially oxidise $5 \mathrm{mC}$ to 5 -hydroxymethylcytosine $(5 \mathrm{hmC})$, which is ultimately excised from DNA, leading to DNA hypomethylation. IDH1/2 enzymes convert a-ketoglutarate to 2-hydroxygluterate, with the latter inhibiting the function of TET enzymes. IDH inhibitors AG-120 (ivosidenib) and AG-221 (enasidenib) reduce the accumulation of 2-hydroxygluterate, relieving inhibition of TET-dependent DNA demethylation, leading to downstream anti-cancer effects.

DNMT, DNA methyltransferase; TET, ten-eleven translocation; IDH, isocitrate dehydrogenase

\section{Therapeutics targeting modifications in histone tails}

Writers responsible for covalent modifications on charged NH2 termini (tails) of histones include histone acetyltransferases (HATs; GCN5, MYST, p300/CBP families) and histone methyltransferases (HMTs; SET [(Su(var)3-9, enhancer of zeste, trithorax)] domain containing and non-SET-domain containing lysinespecific and arginine-specific families). In addition to acetylation and methylation, histone modifications include phosphorylation, ubiquitylation, sumoylation and biotinylation. The type and location of these modifications alters chromatin structure and gene expression. Gene-activating histone modifications include acetylation of lysine 27 in histone $\mathrm{H} 3$, methylation of lysines 4 and 36 in histone $\mathrm{H} 3$, and demethylation of lysine 9 of histone H3. Transcriptionally repressive histone modifications include 
methylation of lysine 9 and 27 in histone $\mathrm{H} 3$ and sumoylation of lysine 59 in histone H4.

Some histone modifications (H3K27 methylation) form docking sites for interactions with polycomb group proteins (PcGs). Once docked, polycomb repressive complex 1 (PRC1) compacts chromatin, causing the physical hinderance of RNA polymerase II, repressing gene transcription. PRC1 has E3 ligase activity, while polycomb repressive complex 2 (PRC2) has HMT activity. One of the best characterised PRC2 subunits is EZH2, which is involved in the methylation of lysine 27 on histone H3. PcG-target genes often contain both repressive (H3K27me3) and active (H3K4me3) modifications [22]. Thus, PcG-target genes exist in a poised ready-to-transcribe state with PcGs holding RNA polymerase II at the transcription start site [22].

HATs (e.g. p300/CBP) are classified into type A (nuclear) and type B (cytoplasmic), depending on whether they acetylate nucleosomal histones or newly translated non-nucleosomal histones, respectively. HATs transfer acetyl groups from acetyl-CoA donors to the amino group of lysine residues of histones. Acetylation of an $\varepsilon$-amino group neutralises the charge of lysine residues, reducing interactions between histones and DNA and making DNA less compact and more accessible to transcription factors. Histone acetylation is associated with gene activation, while deacetylation silences genes. Anti-cancer drugs that target HATs (HAT inhibitors) include the small molecule C646, which selectively inhibits p300/CBP, resulting in reduced acetylation of histone H3 [23]. C646 reduces cell survival and induces cell cycle arrest, mitotic catastrophe and apoptosis $[23,24]$.

Anti-cancer drugs (HMT inhibitors) have also been developed that target the HMTs EZH2, DOT1-like histone lysine methyltransferase (DOT1L), euchromatic histone lysine methyltransferase 2 (G9A), the histone-lysine N-methyltransferase SUV39H1 and protein arginine methyltransferases (PRMT1/3/45/6/8). The EZH2 inhibitor DZNEP reduces H3K27 trimethylation, leading to apoptosis and reduced cell migration (Figure 4) [25]. GSK343 induces apoptosis by increasing caspase-3 and poly ADP-ribose polymerase expression, induces autophagy by inhibiting expression of p62 and suppresses cancer stem cell-like phenotypes [26,27]. GSK126 reduces anti-tumour immunity by increasing the number of myeloid-derived suppressor cells, leading to fewer CD4+ and CD8+ T cells in the tumour microenvironment [28]. DOT1L inhibitors include pinometostat (EPZ5676), which reduces H3K9 methylation and produces a modest clinical response in a subset of adults with advanced acute leukaemia [29], although resistance does occur through drug efflux dependent (ABCB1) and independent mechanisms [30]. The G9A inhibitor BIX01294 inhibits proliferation by downregulating H3K9me1, H3K9me2, H3K27me1 and H3K27me2 modifications, downregulating the anti-apoptotic protein Bcl-2 and upregulating the pro-apoptotic proteins Bax, caspase-3 and caspase-9 [31]. Finally, the SUV39H1 inhibitor chaetocin increases production of reactive oxygen species (ROS), upregulates death-receptor genes and increases expression of CD11b, a surface integrin involved in adhesion interactions with immune cells $[32,33]$.

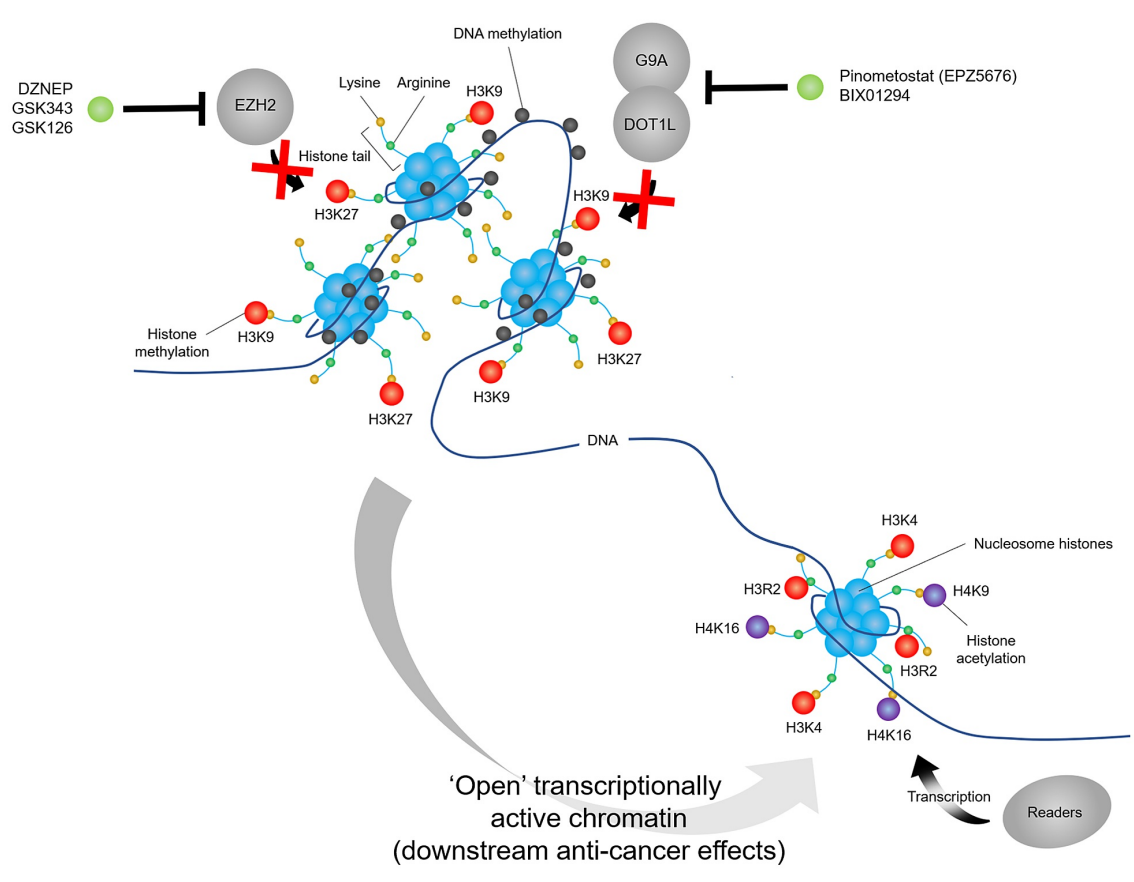

FIGURE 4: Mechanism of action of HMTIs

HMTls inhibit histone methylation by HMTs (e.g. EZH2, DOT1-like histone lysine methyltransferase [DOT1L], euchromatic histone lysine methyltransferase 2 [G9A]), leading to reduction of repressive histone marks (e.g. H3K9 and H3K27 methylation) and adoption of an 'open' transcriptionally active chromatin state with downstream anti-cancer effects.

HMTIs, histone methyltransferase inhibitors; HMT, histone methyltransferase 
Erasers, such as HDACs and histone demethylases (HDMs), remove histone acetyl and methyl groups from histones. HDACs are classified as class I (HDAC1/2/3/8), II (HDAC4/5/6/7/9/10), III or IV. Classes I, II and IV are Zn2+-dependent, while class III is nicotinamide adenine dinucleotide (NAD)-dependent. Dysregulation of HDACs causes a global reduction in histone acetylation, which silences tumour suppressor genes. Anticancer drugs that target HDACs (HDAC inhibitors) include hydroxamates, benzamides, cyclic peptides and fatty acids; many of which target the Zn2+ ion in the HDAC active site. HDAC inhibitors reduce oncogene transcription and signalling, thereby promoting cell cycle arrest and apoptosis (Figure 5). Hydroxamate HDAC inhibitors (belinostat and givinostat) reduce cell survival by causing cell cycle arrest through the induction of p53, induce autophagy and inhibit stemness through the upregulation of differentiation markers (GFAP, Tuj-1) [34,35]. Novel hydroxamate HDAC inhibitors (CG200745, CUDC-101, CUDC-907) reduce cell survival by inducing apoptosis through the downregulation of Hippo pathway proteins and the upregulation of microRNAs (miR-210-3p, miR-509-3p), triggering caspase-dependent degradation of the promyelocytic leukaemia-retinoic acid receptor alpha (PML-RARA) fusion protein and apoptosis in a Mcl-1, Bim and c-Myc dependent manner [36-38]. The benzamide HDAC inhibitor chidamide induces mitochondrial dysfunction, necroptosis and apoptosis [39]. The cyclic peptide HDAC inhibitor romidepsin induces cell cycle arrest and apoptosis by increasing the acetylation of BCL6 [40]. Finally, the fatty acid HDAC inhibitor AR-42 induces cell cycle arrest and apoptosis by inhibiting the AKT/NFKB pathway [41]. Unlike DNMTIs, HDAC inhibitor monotherapy offers low clinical efficacy, achieving poor overall response in AML. However, combination regimens of HDAC inhibitors with DNMTIs, conventional chemotherapy or allogeneic stem cell transplantation have produced encouraging results. Notably, isozyme-selective HDAC inhibitors offer improved safety profiles and comparable efficacy to pan-HDAC agents [42].

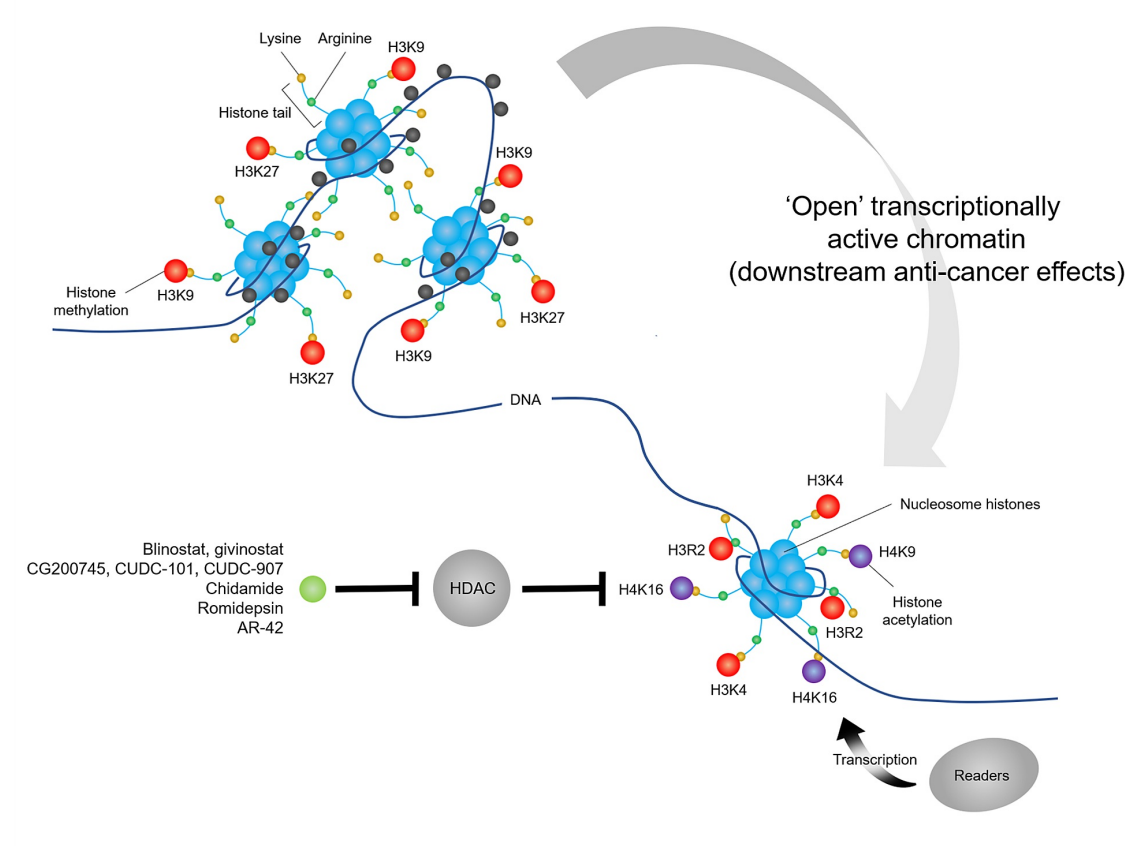

FIGURE 5: Mechanism of action of HDACls

HDACls inhibit the deacetylation of histones by HDACs, leading to an increase in activating histone marks (e.g. H3K9 and H3K16 acetylation) and adoption of an 'open' transcriptionally active chromatin state with downstream anti-cancer effects.

HDACls, histone deacetylase inhibitors; HDAC, histone deacetylase

HDM inhibitor anti-cancer drugs have been developed that target the HDMs lysine demethylase 1A (LSD1/KMD1A) and 5B (KDM5B) as well as ImjC domain-containing proteins. JmjC demethylases are protein hydroxylases involved in free radical-dependent histone modification reactions [43]. LSD1 inhibitors (ORY1001, GSK2879552, tranylcypromine) induce cell cycle arrest and apoptosis by regulating the hexokinase 2 expression and increase the expression of the transcriptional repressor GFI1 as well as the transcription factor PU.1, thus inducing differentiation [44,45]. However, a recent phase I open-label trial of the LSD1 inhibitor GSK2879552 was terminated early due to poor disease control and an unfavourable sideeffect profile [46]. JmjC domain-containing protein inhibitors (GSK-J1, GSK-J4) induce apoptosis and inhibit tumour growth by increasing global levels of repressive trimethylated H3K27 and downregulating cancerpromoting HOX genes $[47,48]$. 


\section{Conclusions}

Cancer epigenetics is a highly complex and rapidly evolving field, with many exciting developments that enhance our understanding of carcinogenesis and disease progression. By examining epigenetic networks, novel therapeutic approaches can be identified that encompass a wide variety of solid and haematological cancers. Therapeutics that normalise or disrupt epigenetic aberrations hold promise across several malignancies, with well-defined clinical efficacy established in distinct clinical settings. Considering the abundance of epigenetic targets and agents in development, a systematic approach for the identification and validation of potential drug targets is essential to optimise drug development and translate the promise of upcoming epigenetic agents to routine patient management. Future research should focus on achieving a deeper understanding of epigenetic mechanisms to yield better therapies as well as exploiting therapeutics that promote global epigenetic normalisation to counteract epigenetic aberrations. These approaches will enhance the utility of epigenetic drugs, maximising benefits in terms of returns of research investment and alleviating the burden of cancer on public health.

\section{Additional Information \\ Disclosures}

Conflicts of interest: In compliance with the ICMJE uniform disclosure form, all authors declare the following: Payment/services info: All authors have declared that no financial support was received from any organization for the submitted work. Financial relationships: All authors have declared that they have no financial relationships at present or within the previous three years with any organizations that might have an interest in the submitted work. Other relationships: All authors have declared that there are no other relationships or activities that could appear to have influenced the submitted work.

\section{References}

1. Leading causes of death, UK: 2001 to 2018. (2020). Accessed: August 27, 2020: https://www.ons.gov.uk/peoplepopulationandcommunity/healthandsocialcare/causesofdeath/articles/leadingcausesofdeathı

2. Cancer Statistics for the UK. (2020). Accessed: August 27, 2020: https://www.cancerresearchuk.org/healthprofessional/cancer-statistics-for-the-uk.

3. Falahi F, van Kruchten M, Martinet N, Hospers GA, Rots MG: Current and upcoming approaches to exploit the reversibility of epigenetic mutations in breast cancer. Breast Cancer Res. 2014, 29:412. 10.1186/s13058014-0412-z

4. Azacitidine for the treatment of myelodysplastic syndromes, chronic myelomonocytic leukaemia and acute myeloid leukaemia. (2020). Accessed: August 27, 2020: https://www.nice.org.uk/guidance/ta218/chapter/1Guidance.

5. Panobinostat for treating multiple myeloma after at least 2 previous treatments . (2020). Accessed: August 27, 2020: https://www.nice.org.uk/guidance/TA380.

6. Public summary of opinion on orphan designation. Tazemetostat for the treatment of diffuse large B-cell lymphoma. (2018). Accessed: August 27, 2020: https://www.ema.europa.eu/en/documents/orphandesignation/eu/3/18/2004-public-summary-opinion-orphan-designation-taze....

7. Bates SE: Epigenetic therapies for cancer. N Engl J Med. 2020, 13:650-663. 10.1056/NEJMra1805035

8. Li Y, Li Z, Zhu WG: Molecular mechanisms of epigenetic regulators as activatable targets in cancer theranostics. Curr Med Chem. 2019, 26:1328-1350. 10.2174/0929867324666170921101947

9. Angeloni A, Bogdanovic O: Enhancer DNA methylation: implications for gene regulation. Essays Biochem. 2019, 63:707-715. 10.1042/EBC20190030

10. Schaefer M, Hagemann S, Hanna K, Lyko F: Azacytidine inhibits RNA methylation at DNMT2 target sites in human cancer cell lines. Cancer Res. 2009, 15:8127-8132. 10.1158/0008-5472.CAN-09-0458

11. Gowher H, Jeltsch A: Mechanism of inhibition of DNA methyltransferases by cytidine analogs in cancer therapy. Cancer Biology \& Therapy. 2004, 12:1062-1068. 10.4161/cbt.3.11.1308

12. Dan H, Zhang S, Zhou Y, Guan Q: DNA methyltransferase inhibitors: catalysts for antitumour immune responses. Onco Targets Ther. 2019, 12:10903-10916. 10.2147/OTT.S217767

13. Scourzic L, Mouly E, Bernard OA: TET proteins and the control of cytosine demethylation in cancer . Genome Med. 2015, 7:9. 10.1186/s13073-015-0134-6

14. Cancer Genome Atlas Research Network, Ley TJ, Miller C, et al.: Genomic and epigenomic landscapes of adult de novo acute myeloid leukemia. N Engl J Med. 2013, 30:2059-2074. 10.1056/NEJMoa1301689

15. Figueroa ME, Abdel-Wahab O, Lu C, et al.: Leukemic IDH1 and IDH2 mutations result in a hypermethylation phenotype, disrupt TET2 function, and impair hematopoietic differentiation. Cancer Cell. 2010, 14:553-567. 10.1016/j.ccr.2010.11.015

16. Liu X, Gong Y: Isocitrate dehydrogenase inhibitors in acute myeloid leukemia . Biomark Res. 2019, 22:22. 10.1186/s40364-019-0173-z

17. Ward PS, Patel J, Wise DR, et al.: The common feature of leukemia-associated IDH1 and IDH2 mutations is a neomorphic enzyme activity converting alpha-ketoglutarate to 2-hydroxyglutarate. Cancer Cell. 2010, 16:225-234. 10.1016/j.ccr.2010.01.020

18. Yang H, Liu Y, Bai F, et al.: Tumor development is associated with decrease of TET gene expression and 5methylcytosine hydroxylation. Oncogene. 2013, 31:663-669. 10.1038/onc.2012.67

19. Yen K, Travins J, Wang F, et al.: AG-221, a first-in-class therapy targeting acute myeloid leukemia harboring oncogenic IDH2 mutations. Cancer Discov. 2017, 7:478-493. 10.1158/2159-8290.CD-16-1034

20. DiNardo CD, Stein EM, de Botton S, et al.: Durable remissions with ivosidenib in IDH1-mutated relapsed or refractory AML. N Engl J Med. 2018, 21:2386-2398. 10.1056/NEJMoa1716984

21. Stein EM, DiNardo CD, Pollyea DA, et al.: Enasidenib in mutant IDH2 relapsed or refractory acute myeloid leukemia. Blood. 2017, 10:722-731. 10.1182/blood-2017-04-779405

22. Messier TL, Boyd JR, Gordon JA, Stein JL, Lian JB, Stein GS: Oncofetal epigenetic bivalency in BC CElls: H3K4 and H3K27 tri-methylation as a biomarker for phenotypic plasticity. J Cell Physiol. 2016, 231:2474- 
2481. 10.1002/jcp.25359

23. Wang YM, Gu ML, Meng FS, Jiao WR, Zhou XX, Yao HP, Ji F: Histone acetyltransferase p300/CBP inhibitor C646 blocks the survival and invasion pathways of gastric cancer cell lines. Int J Oncol. 2017, 51:1860-1868. 10.3892/ijo.2017.4176

24. Oike T, Komachi M, Ogiwara H, et al.: C646, a selective small molecule inhibitor of histone acetyltransferase p300, radiosensitizes lung cancer cells by enhancing mitotic catastrophe. Radiother Oncol. 2014, 111:222227. 10.1016/j.radonc.2014.03.015

25. Girard N, Bazille C, Lhuissier E, Benateau H, Llombart-Bosch A, Boumediene K, Bauge C: 3Deazaneplanocin A (DZNep), an inhibitor of the histone methyltransferase EZH2, induces apoptosis and reduces cell migration in chondrosarcoma cells. PLoS One. 2014, 22:98176. 10.1371/journal.pone.0098176

26. Xiong X, Zhang J, Li A, et al.: GSK343 induces programmed cell death through the inhibition of EZH2 and FBP1 in osteosarcoma cells. Cancer Biol Ther. 2020, 21:213-222. 10.1080/15384047.2019.1680061

27. Yu T, Wang Y, Hu Q, et al.: The EZH2 inhibitor GSK343 suppresses cancer stem-like phenotypes and reverses mesenchymal transition in glioma cells. Oncotarget. 2017, 27:98348-98359. 10.18632/oncotarget.21311

28. Huang S, Wang Z, Zhou J, et al.: EZH2 inhibitor GSK126 suppresses antitumor immunity by driving production of myeloid-derived suppressor cells. Cancer Res. 2019, 15:2009-2020. 10.1158/0008-5472.CAN18-2395

29. Stein EM, Garcia-Manero G, Rizzieri DA, et al.: The DOT1L inhibitor pinometostat reduces H3K79 methylation and has modest clinical activity in adult acute leukemia. Blood. 2018, 14:2661-2669. 10.1182/blood-2017-12-818948

30. Campbell CT, Haladyna JN, Drubin DA, et al.: Mechanisms of pinometostat (EPZ-5676) treatment-emergent resistance in MLL-rearranged leukemia. Mol Cancer Ther. 2017, 16:1669-1679. 10.1158/1535-7163.mct-160693

31. Guo AS, Huang YQ, Ma XD, Lin RS: Mechanism of G9a inhibitor BIX-01294 acting on U251 glioma cells . Mol Med Rep. 2016, 14:4613-4621. 10.3892/mmr.2016.5815

32. Chaib H, Nebbioso A, Prebet T, et al.: Anti-leukemia activity of chaetocin via death receptor-dependent apoptosis and dual modulation of the histone methyl-transferase SUV39H1. Leukemia. 2012, 26:662-674. 10.1038/leu.2011.271

33. Lai YS, Chen JY, Tsai HJ, Chen TY, Hung WC: The SUV39H1 inhibitor chaetocin induces differentiation and shows synergistic cytotoxicity with other epigenetic drugs in acute myeloid leukemia cells. Blood Cancer J. 2015, 15:313. 10.1038/bcj.2015.37

34. Lu P, Gu Y, Li L, Wang F, Yang X, Yang Y: Belinostat suppresses cell proliferation by inactivating Wnt/ $\beta$ catenin pathway and promotes apoptosis through regulating PKC pathway in breast cancer. Artif Cells Nanomed Biotechnol. 2019, 47:3955-3960. 10.1080/21691401.2019.1671855

35. Marampon F, Leoni F, Mancini A, et al.: Histone deacetylase inhibitor ITF2357 (givinostat) reverts transformed phenotype and counteracts stemness in in vitro and in vivo models of human glioblastoma. J Cancer Res Clin Oncol. 2019, 145:393-409. 10.1007/s00432-018-2800-8

36. Jung DE, Park SB, Kim K, Kim C, Song SY: CG200745, an HDAC inhibitor, induces anti-tumour effects in cholangiocarcinoma cell lines via miRNAs targeting the Hippo pathway. Sci Rep. 2017, 7:10921. 10.1038/s41598-017-11094-3

37. Zhang T, Ma D, Wei D, et al.: CUDC-101 overcomes arsenic trioxide resistance via caspase-dependent promyelocytic leukemia-retinoic acid receptor alpha degradation in acute promyelocytic leukemia. Anticancer Drugs. 2020, 31:158-168. 10.1097/CAD.0000000000000847

38. Li X, Su Y, Madlambayan G, et al.: Antileukemic activity and mechanism of action of the novel PI3K and histone deacetylase dual inhibitor CUDC-907 in acute myeloid leukemia. Haematologica. 2019, 104:22252240. 10.3324/haematol.2018.201343

39. Chi Z, Gao H, Liu H, Wu B, Zhang B, Gu M, Yang W: Chidamide induces necroptosis via regulation of c-FLIPL expression in Jurkat and HUT-78 cells. Mol Med Rep. 2020, 21:936-944. 10.3892/mmr.2019.10873

40. Cortiguera MG, García-Gaipo L, Wagner SD, León J, Batlle-López A, Delgado MD: Suppression of BCL6 function by HDAC inhibitor mediated acetylation and chromatin modification enhances BET inhibitor effects in B-cell lymphoma cells. Sci Rep. 2019, 11:16495. 10.1038/s41598-019-52714-4

41. Duan S, Gong X, Liu X, et al.: Histone deacetylase inhibitor, AR-42, exerts antitumor effects by inducing apoptosis and cell cycle arrest in Y79 cells. J Cell Physiol. 2019, 234:22411-22423. 10.1002/jcp.28806

42. Sun Y, Chen BR, Deshpande A: Epigenetic regulators in the development, maintenance, and therapeutic targeting of acute myeloid leukemia. Front Oncol. 2018, 8:41. 10.3389/fonc.2018.00041

43. Jambhekar A, Anastas JN, Shi Y: Histone lysine demethylase inhibitors. Cold Spring Harb Perspect Med. 2017, 7:026484. 10.1101/cshperspect.a026484

44. Lu Z, Guo Y, Zhang X, Li J, Li L, Zhang S, Shan C: ORY-1001 suppresses cell growth and induces apoptosis in lung cancer through triggering HK2 mediated Warburg effect. Front Pharmacol. 2018, 4:1411. 10.3389/fphar.2018.01411

45. Barth J, Abou-El-Ardat K, Dalic D, et al.: LSD1 inhibition by tranylcypromine derivatives interferes with GFI1-mediated repression of PU.1 target genes and induces differentiation in AML. Leukemia. 2019, 33:1411-1426. 10.1038/s41375-018-0375-7

46. Bauer TM, Besse B, Martinez-Marti A, et al.: Phase I, open-label, dose-escalation study of the safety, pharmacokinetics, pharmacodynamics, and efficacy of GSK2879552 in relapsed/refractory SCLC. J Thorac Oncol. 2019, 14:1828-1838. 10.1016/j.jtho.2019.06.021

47. Zhang W, Cheng J, Diao P, Wang D, Zhang W, Jiang H, Wang Y: Therapeutically targeting head and neck squamous cell carcinoma through synergistic inhibition of LSD1 and JMJD3 by TCP and GSK-J1. Br J Cancer. 2019, 122:528-538. 10.1038/s41416-019-0680-6

48. Li Y, Zhang M, Sheng M, et al.: Therapeutic potential of GSK-J4, a histone demethylase KDM6B/JMJD3 inhibitor, for acute myeloid leukemia. J Cancer Res Clin Oncol. 2018, 144:1065-1077. 10.1007/s00432-018$2631-7$ 